\title{
Association of red cell distribution width with all-cause and cardiovascular-specific mortality in African American and white adults: a prospective cohort study
}

\author{
Salman M. Tajuddin ${ }^{1}$, Mike A. Nalls ${ }^{2,3}$, Alan B. Zonderman ${ }^{1}$ and Michele K. Evans ${ }^{1 *}$
}

\begin{abstract}
Background: While the mortality rate is declining in the United States, the life expectancy gap among different population groups suggests a need to identify biomarkers to improve early identification of individuals at risk. Red cell distribution width (RDW), a measure of anisocytosis, is an emerging biomarker of chronic disease morbidity and mortality, particularly in the elderly. However, little is known about its association with mortality risk in younger adults. The objectives of this study were to investigate the association between RDW and overall and cause-specific mortality risk, and to identify novel determinants of RDW level.
\end{abstract}

Methods: We used prospectively collected data from the Healthy Aging in Neighborhoods of Diversity across the Life Span study conducted in Baltimore, Maryland. At baseline (2004-2009), the study recruited 3720 African American and white men and women aged 30-64 years. Participants provided peripheral venous blood for RDW measurement as part of complete blood count, and genotyping. Mortality status was ascertained using the National Death Index database through December 31, 2013. Multivariable adjusted Cox proportional hazards regression models were fitted to assess mortality risk, and multiple linear regression models to identify determinants of RDW level.

Results: Participants' mean age was 48.1 (9.2) years. Of 2726 participants included in the present analyses, 57\% were African Americans, and 56\% were women. After 18,424 person-years of follow-up time, there were 226 deaths, and the leading cause of death were cardiovascular diseases (31.9\%). Participants in the highest quartile of RDW had a 1.73fold increased all-cause mortality risk (highest quartile vs. lowest quartile, multivariable adjusted hazard ratio $=1.73$, $95 \%$ confidence interval: $1.10-2.74, \mathrm{p}$-trend $=0.006$ ). This effect was significantly modified by body mass index (p-interaction $=0.004$ ). Similar risk was observed for cardiovascular disease-specific mortality. Independent of body mass index, waist-hip ratio and illicit drug use were significantly associated with RDW.

Conclusions: Elevated RDW was associated with a substantial risk of all-cause and cardiovascular disease-specific mortalities that was modified by body mass index. Central obesity and illicit drug use influence RDW level. In vulnerable populations at-risk for health disparities, RDW could provide a useful and inexpensive biomarker of mortality.

Keywords: Red cell distribution width, Mortality biomarker, Premature mortality, Cardiovascular disease, Survival analysis, African Americans, Whites

\footnotetext{
*Correspondence: EvansM@grc.nia.nih.gov

${ }^{1}$ Laboratory of Epidemiology and Population Sciences, National Institute

on Aging, National Institutes of Health, Room \# 04C222, Suite 100, 251

Bayview Boulevard, Baltimore, MD 21224, USA

Full list of author information is available at the end of the article
} 


\section{Background}

Red cell distribution width (RDW), a measure of variation in red blood cell size also known as anisocytosis, is an emerging biomarker of chronic disease morbidity and mortality [1]. RDW is associated with increased risk of cardiovascular diseases (CVD) including coronary artery disease, heart failure, atherosclerosis, and venous thromboembolism independent of traditional CVD risk factors [2-7]. Elevated RDW was also associated with overall and disease-specific mortality risk [6, 8-11]. Most of these studies, however, were conducted in older individuals, and hence the role of RDW on mortality risk in younger individuals is poorly understood. Although the specific biological mechanisms behind the association between RDW and adverse health outcomes have not been fully explained, it was suggested that it could be mediated through inflammatory cytokines, or oxidative stress $[2,9,12]$. RDW is influenced by factors such as sex, race, body mass index (BMI), cigarette smoking, markers of systemic inflammation and lipids although results were inconclusive $[6,7,12,13]$. Whereas genetic variants in loci including G6PD, CD36, and NOL4L have been linked with RDW level [14, 15], the interplay between environmental factors and genetic variants on RDW has not yet been investigated.

While the age-adjusted mortality rate in the United States has been declining, there remains a gap in premature mortality and life expectancy among racial/ethnic groups and individuals of various socioeconomic status $[16,17]$. Besides, Case and Deaton recently reported that in the past $10-15$ years there has been an alarmingly increasing mortality rate among white American men and women aged 30-54 years [18]. Established risk factors of mortality include tobacco use, obesity, and clinical markers such as elevated blood pressure and hyperlipidemia [19]. The growing mortality disparity and widening of life expectancy gap suggest a need to identify additional biomarkers to improve early detection of at-risk individuals to enhance precise and targeted interventions.

This new evidence of sharply declining longevity not only among African Americans (AA) but also young whites has made us reevaluate the role of RDW in younger populations of urban AA and whites since RDW was shown to be a good predictor of mortality for those in middle and old age [11]. Using prospectively collected data (August 2004-December 2013) from the Healthy Aging in Neighborhoods of Diversity across the Life Span (HANDLS) study, which is a large longitudinal study of socioeconomically diverse community-dwelling AA and white working-age adults, the objectives of this study were (1) to assess the association between RDW and allcause and cause-specific mortality; (2) to identify lifestyle and environmental determinants of RDW; and (3) to explore the effect of gene $\times$ environment interaction on RDW.

\section{Methods \\ Population and study design}

The HANDLS study is a large population-based prospective longitudinal study conducted in Baltimore, MD. Study design and methods of data collection have been described previously [20]. Briefly, the HANDLS study was designed to establish a single-site study for the investigation of age-related health disparities in socioeconomically diverse adult AAs and whites. The initial wave of data collection recruited 3720 individuals from 2004 to 2009. Participants were men and women aged $30-64$ years, above and below $125 \%$ of the 2004 US Federal poverty level. At baseline, 2744 participants had complete RDW data. We excluded 18 subjects with sickle cell disease leaving 2726 for the current analysis.

\section{Red cell distribution width and covariates}

During the baseline data collection (2004-2009), study participants were interviewed using structured questionnaires, underwent medical examinations, and provided social and medical histories as well as a blood sample. Data included age, sex, race, poverty status, BMI ( $\mathrm{kg} /$ $\mathrm{m}^{2}$ ), waist-hip ratio (WHR), current smoking status, current alcohol use, highest education level attained and reported illicit drug use (e.g., marijuana, cocaine). RDW was measured by automated Coulter DXH 800 hematology analyzer as part of peripheral complete blood count (Beckman Coulter, Brea, CA), and was expressed as coefficient of variation (\%) of red blood cell volume distribution. Regular calibration was performed every 3 months on the hematology analyzer and quality control was performed according to the manufacturer's recommendations. Hypertension was defined as self-reported history of hypertension, use of anti-hypertensive medications, or blood pressure of $>140 / 90$. Diabetes mellitus was defined as self-reported history of Type I or II diabetes mellitus, reported use of diabetes medication, or fasting plasma glucose $\geq 126 \mathrm{mg} / \mathrm{dl}$. Self-reported depressive symptoms were assessed using the Center for Epidemiologic Studies-Depression (CES-D) scale [21]. Additional covariates included hemoglobin, serum iron, serum vitamin B12, total white blood cell (WBC) count, erythrocyte sedimentation rate (ESR), high-sensitivity C-reactive protein (hsCRP), low density lipoprotein-cholesterol (LDL), and estimated glomerular filtration rate (eGFR) [22].

\section{Mortality and cause of death assessment}

Mortality was ascertained through the National Death Index (NDI) database. Each study participant was 
matched to the NDI using name, date and state of birth, sex, race, maiden name and social security number $[16$, 23]. Participants were followed from the date of study enrolment to date of death or censored date, December 31, 2013. Primary cause of death is based on the International Classification of Diseases 10th edition (ICD-10). Death due to cardiovascular diseases included the ICD codes from I10.0 to I82.9. A total of 2704 participants had complete follow-up and mortality outcome data.

\section{Genotyping}

A subset of AA participants was genotyped using Illumina $1 \mathrm{M}$ and Illumina $1 \mathrm{M}$-Duo genotyping arrays (Illumina, San Diego, CA). Genotype calling and quality control followed standard procedures. Samples with call rate $<95 \%$, sex mismatch, ethnic ancestry outliers and cryptic related individuals were excluded. SNPs with minor allele frequency less than 1\%, Hardy-Weinberg equilibrium $p$ value of less than $1.0 \times 10^{-7}$, call rate $<95 \%$ were excluded. After sample and genotype quality control, 1024 AAs had complete genome-wide genotype data. Genotype data management and quality control was performed using PLINK (https://www.cog-genomics.org/plink2) [24]. Principal components were generated using a set of linkage disequilibrium based pruned independent single nucleotide polymorphisms. This set of single nucleotide polymorphisms were selected under PLINK default settings of pairwise correlation coefficient of 0.2 between single nucleotide polymorphisms in a sliding window of size of 50 . The correlation coefficient of 0.2 was used to select near completely independent SNPs to avoid collinearity between them. To increase power, genotypes were imputed using the 1000 Genomes Project phase 1 version 3 reference panel. Genotypes were phased using the $\mathrm{MaCH}$ software and imputation was performed using minimac software (http://genome.sph. umich.edu/wiki/MaCH).

\section{Statistical analysis}

Distributions of continuous variables were plotted using histograms and were checked for skewness. To achieve normal distribution, hsCRP, ESR, and WBC count were natural-logarithm transformed. Categorical variables included in the analysis were: sex, race (AA/white), poverty status (above/below), college degree (yes/no), current cigarette smoking (yes/no), current alcohol use (yes/ no), hypertension (yes/no), diabetes mellitus (yes/no), CES-D score $(<16 / \geq 16)$, marijuana use (yes/no), current cocaine use (yes/no).

\section{Survival analysis}

We fitted unadjusted and multivariable adjusted Cox proportional hazards regression models to estimate hazard ratios and 95\% confidence intervals (CI) of allcause mortality and CVD-specific mortality. Age at study entrance and exit (death or censored date) were used as the measurement of follow-up time [25]. To allow a balanced comparison of mortality risk across the different strata, RDW was categorized by quartiles (cut-offs: $13.2,13.8$, and $14.6 \%$ ). We initially adjusted for age, sex, race, and poverty status. The full Cox regression model was also adjusted for established predictors of mortality and potential confounders: current cigarette smoking, BMI, LDL, hypertension and diabetes mellitus. Test for linear trend across the categories of RDW was performed by assigning the median value to each quartile of RDW and included this variable as a continuous term in the full Cox regression model. The proportional hazards assumption was checked by inspection of loglog survival curves, and formally evaluated using the scaled Schoenfeld residuals method yielding p-values $>0.1$ suggesting the proportional hazards assumptions were not violated. Although for the sake of parsimony we adjusted the full Cox regression model for the above nine variables, we performed sensitivity analyses by adding the following variables one at time to the full model, and simultaneously adjusting for them: WHR, systolic blood pressure, hemoglobin, serum iron, serum vitamin B12, ESR, hsCRP, WBC count, and eGFR.

To assess the association between sickle cell trait (SCT) and RDW and its role on the association between RDW and mortality risk, we used $H B B$-rs334 $(A>T$, p.Glu6Val, minor allele frequency $=6.2 \%$ ) to determine SCT status among study participants. We compared RDW level between non-carriers and carriers of the sickle cell allele. Main effects of SCT as well as interaction between RDW and SCT on all-cause and CVD-specific mortality risk were estimated.

We explored effect modifications by established predictors of mortality by including a multiplicative interaction term in the full Cox regression model. The variables tested for interaction were age, sex, race, poverty status, current cigarette smoking, BMI, LDL, hypertension, and diabetes mellitus. Evidence of interaction was tested by comparing the models with and without the interaction term using a likelihood ratio test.

To assess the performance of RDW as predictive marker of all-cause and CVD-specific mortality we assessed model calibration of the fully adjusted Cox regression models using the modified Nam-D'Agostino goodness-of-fit (GOF) test for survival data [26].

\section{Determinants of red cell distribution width}

To identify determinants of RDW, we fitted multiple linear regression models to estimate beta coefficients and 95\% CIs. The initial model included age, sex, race, and 
poverty status; and the full model was further adjusted for potential confounders and previously reported determinants of RDW [5, 6] including smoking status, BMI, hypertension, diabetes mellitus, LDL, eGFR, and hsCRP. Sensitivity analyses were performed by further adjusting the full model for hemoglobin levels.

\section{Gene $x$ environment interaction analysis in RDW}

Among AAs $(\mathrm{N}=998)$ who had complete RDW and genotype data, gene $\times$ environment interactions were assessed between lifestyle factors and genetic variants by including an interaction term into the regression model adjusted for age, sex and the first five principal components to account for population stratifications. The variants tested were: TMEM57-RHD-rs10903129, NOL4L-rs4911241, CD36-rs3211938, LINC01184SLC12A2-rs10063647, LINC01184-SLC12A2-rs10089, TRIB1-rs2954029 [14, 15]. These variants were selected since they were identified by one of the largest genetic association studies and may have biological significance in red cell biology. All statistical tests were two-sided, and p-value $<0.05$ was considered significant. Data analyses were performed using the $\mathrm{R}$ statistical software version 3.2.3 (https://www.R-project.org/) [27].

\section{Results}

The characteristics of the 2726 study participants in the present analyses stratified by race are shown in Table 1: $57 \%$ were AAs, $56 \%$ were women, and baseline mean age (standard deviation) was 48.1 (9.2) years. The mean (standard deviation) of RDW was $14.1 \%$ (1.7).

\section{Association of RDW with all-cause mortality}

Participants were followed for up to 9.4 years (median follow-up time $=6.8$ years). After 18,424 person-years of follow-up time, there were a total of 226 deaths. CVD was the leading cause death in our cohort [72/226 (31.9\%)] (Table 1). The unadjusted hazard ratio (HR) for all-cause mortality among participants in the highest quartile of RDW was 1.90 (95\% CI 1.30-2.78, p-trend < 0.001) compared to those in the lowest quartile. After adjusting for age, sex, race and poverty status, the risk of allcause mortality for people in the highest RDW quartile was 1.95 (95\% CI 1.32-2.88, p-trend < 0.001). In the full model adjusted for age, sex, race, poverty status, smoking status, BMI, LDL, hypertension and diabetes mellitus, participants in the highest quartile had a $73 \%$ increased risk of all-cause mortality (95\% CI 1.10-2.74, $\mathrm{p}$-trend $=0.006$ ) (Table 2). To identify subgroup effects of RDW on all-cause mortality risk, we performed stratified analyses. The results of stratified analysis by sex, race, poverty status, current smoking and BMI for
Table 1 Participant characteristics and mortality outcomes stratified by race

\begin{tabular}{|c|c|c|c|c|}
\hline \multirow[t]{2}{*}{ Characteristics } & \multicolumn{2}{|c|}{ African Americans } & \multicolumn{2}{|l|}{ Whites } \\
\hline & $N=1563$ & $\%$ & $N=1163$ & $\%$ \\
\hline Age, mean (SD) & $48.0(9.2)$ & & $48.2(9.3)$ & \\
\hline \multicolumn{5}{|l|}{ Sex } \\
\hline Women & 880 & 56.3 & 658 & 56.6 \\
\hline Men & 683 & 43.7 & 505 & 43.4 \\
\hline \multicolumn{5}{|l|}{ Poverty status } \\
\hline Above & 823 & 52.7 & 788 & 67.8 \\
\hline Below & 740 & 47.3 & 375 & 32.2 \\
\hline \multicolumn{5}{|c|}{ Education-college degree } \\
\hline No & 1458 & 93.8 & 913 & 82.9 \\
\hline Yes & 97 & 6.2 & 188 & 17.1 \\
\hline Missing & 8 & & 62 & \\
\hline \multicolumn{5}{|l|}{ Current smoking status } \\
\hline No & 686 & 49.9 & 574 & 54.4 \\
\hline Yes & 688 & 50.1 & 481 & 45.6 \\
\hline Missing & 189 & & 108 & \\
\hline \multicolumn{5}{|l|}{ Current alcohol use } \\
\hline No & 597 & 43.6 & 426 & 40.3 \\
\hline Yes & 773 & 56.4 & 630 & 59.7 \\
\hline Missing & 193 & & 107 & \\
\hline \multicolumn{5}{|l|}{ Marijuana use } \\
\hline No & 1141 & 83.3 & 952 & 90.2 \\
\hline Yes & 228 & 16.7 & 104 & 9.8 \\
\hline Missing & 194 & & 107 & \\
\hline \multicolumn{5}{|l|}{ Current cocaine use } \\
\hline No & 1271 & 92.4 & 1016 & 95.8 \\
\hline Yes & 104 & 7.6 & 45 & 4.2 \\
\hline Missing & 188 & & 102 & \\
\hline \multicolumn{5}{|l|}{ CES-D scale score } \\
\hline$<16$ & 859 & 58.9 & 661 & 59 \\
\hline$\geq 16$ & 600 & 41.1 & 460 & 41 \\
\hline Missing & 104 & & 42 & \\
\hline \multicolumn{5}{|l|}{ Hypertension } \\
\hline No & 712 & 48.6 & 660 & 58.9 \\
\hline Yes & 752 & 51.4 & 461 & 41.1 \\
\hline Missing & 99 & & 42 & \\
\hline \multicolumn{5}{|l|}{ Diabetes mellitus } \\
\hline No & 1209 & 81.9 & 937 & 83.3 \\
\hline Yes & 268 & 18.1 & 188 & 16.7 \\
\hline Missing & 86 & & 38 & \\
\hline RDW (\%) & $14.48(1.91)$ & & $13.80(1.32)$ & \\
\hline BMI $\left(\mathrm{kg} / \mathrm{m}^{2}\right)$ & $29.95(7.97)$ & & $30.21(7.77)$ & \\
\hline $\mathrm{LDL}(\mathrm{mg} / \mathrm{dL})$ & $107.16(37.13)$ & & $111.94(35.98)$ & \\
\hline $\mathrm{eGFR}\left(\mathrm{ml} / \mathrm{min} / 1.73 \mathrm{~m}^{2}\right)$ & $102.51(22.72)$ & & $96.14(17.72)$ & \\
\hline Total WBC $\left(10^{9} / \mathrm{L}\right)$ & $6.18(2.04)$ & & $7.04(2.28)$ & \\
\hline hsCRP (mg/L) & $5.47(12.10)$ & & $4.45(6.62)$ & \\
\hline WHR & $0.91(0.08)$ & & $0.95(0.39)$ & \\
\hline
\end{tabular}


Table 1 continued

\begin{tabular}{llllll}
\hline Characteristics & \multicolumn{2}{l}{ African Americans } & & Whites & \\
\cline { 2 - 2 } \cline { 5 - 6 } \cline { 5 - 6 } & $\mathbf{N}=\mathbf{1 5 6 3}$ & $\mathbf{N}=1163$ & $\%$ \\
\hline All-cause mortality & 141 & & 85 & \\
CVD-specific mortality & 48 & & & \\
\hline
\end{tabular}

$B M I$ body mass index, CES-D Center for Epidemiologic Studies Depression Scale, hsCRP high-sensitivity C-reactive protein, CVD cardiovascular disease, eGFR estimated glomerular filtration rate, $L D L$ low density lipoprotein cholesterol, $R D W$ red cell distribution width, SD standard deviation, WBC white blood cell count, WHR waist-hip ratio

all-cause mortality are presented in Table 3 . We observed a statistically significant interaction between RDW and BMI (p-interaction $=0.004)$. The increased risk of death associated with higher RDW level was stronger among individuals with $\mathrm{BMI} \geq 25.0$ (adjusted HR for the highest quartile vs. lowest quartile $=2.06,95 \%$ CI $1.11-3.83$, $\mathrm{p}$-trend $=0.004)$. There was no effect modification by the other variables considered ( $\mathrm{p}$-interaction $>0.05$ ). Results from the sensitivity analyses after controlling for additional covariates including hemoglobin, serum iron and serum vitamin $B_{12}$ were consistent with the main results from the full Cox proportional hazards regression model.

\section{Association of RDW with CVD-specific mortality}

Similarly, higher RDW level was associated with an increased risk of CVD-specific mortality. In the multivariable adjusted model, HR for the highest quartile was 2.49 (95\% CI 1.03-6.05, p-trend $=0.004)$ (Table 2). The increased risk of CVD-specific mortality associated with higher RDW was more pronounced among participants with $\mathrm{BMI} \geq 25$ (p-interaction $=0.05)$ (Additional file 1 : Table S1).

SCT was not associated with all-cause and CVD-specific mortality. There was also no effect modification or confounding by SCT on the association between RDW and increased mortality risk. Similarly, RDW levels did not differ between non-carriers and carriers of the sickle cell allele (data not shown). Risk estimates of BMI and LDL on all-cause mortality and CVD-specific mortality are shown in Additional file 1: Table S2.

Results of model calibration of RDW in the fully adjusted Cox regression model was $P_{G O F}=0.445$ $\left(\mathrm{X}^{2}=3.718, \mathrm{df}=4\right)$ for all-cause mortality, and $P_{G O F}=0.965\left(x^{2}=0.071, \mathrm{df}=2\right)$ for CVD-specific mortality indicating that the models were not miscalibrated (Additional file 1: Figure S1).

\section{Determinants of RDW}

\section{Replication of previously identified RDW determinants}

We replicated known predictors of RDW (Additional file 1: Table S3). In the full multivariable adjusted linear regression model, RDW was significantly higher among AAs compared to whites [beta (b) $(95 \% \mathrm{CI})=0.6(0.5$, $0.8)]$, and current smokers compared to non-current smokers $[0.3(0.1,0.4)]$, while RDW was lower among men compared to women $[-0.3(-0.5,-0.2)]$. There was a direct association between RDW and BMI, and hsCRP. On the other hand, LDL $[-0.005(-0.007,-0.003)]$, and

Table 2 Risk of all-cause and cardiovascular disease-specific mortality and red cell distribution width

\begin{tabular}{llllll}
\hline RDW & Total & Events & $\left.\mathbf{H R}^{\mathbf{b}} \mathbf{( 9 5 \%} \mathbf{C l}\right)$ & $\mathbf{H R}^{\mathbf{c}} \mathbf{( 9 5 \% \mathbf { C l } )}$ & $\left.\mathbf{H R}^{\mathbf{d}} \mathbf{( 9 5 \%} \mathbf{C l}\right)$ \\
\hline All-cause mortality & & & & & 1.00 \\
RDW-Q1 & 638 & 41 & 1.00 & $1.17(0.78-1.76)$ & 1.00 \\
RDW-Q2 & 743 & 56 & $1.19(0.79-1.78)$ & $1.13(0.74-1.71)$ & $1.06(0.66-1.72)$ \\
RDW-Q3 & 647 & 50 & $1.12(0.74-1.7)$ & $1.95(1.32-2.88)$ & $1.08(0.67-1.75)$ \\
RDW-Q4 & 676 & 79 & $1.90(1.30-2.78)$ & $<0.001$ & $1.73(1.10-2.74)$ \\
P-trend & & & $<0.001$ & & 0.006 \\
CVD-specific mortality & & & & 1.00 & 1.00 \\
RDW-Q1 & 605 & 15 & 1.00 & $1.55(0.65-3.67)$ & $0.92(0.34-2.49)$ \\
RDW-Q2 & 702 & 16 & $1.60(0.68-3.78)$ & $1.73(0.73-4.09)$ & $1.09(0.41-2.85)$ \\
RDW-Q3 & 613 & 33 & $1.79(0.77-4.18)$ & $3.89(1.75-8.62)$ & $2.49(1.03-6.05)$ \\
RDW-Q4 & 630 & & $4.03(1.86-8.74)$ & $<0.001$ & 0.004 \\
P-trend & & & $<0.001$ & \\
\hline
\end{tabular}

Cl confidence interval, CVD cardiovascular diseases, $H R$ hazard ratio

a RDW, red-cell distribution level in quartiles. Quartile cut-off points were 13.2, 13.8, and $14.6 \%$

b Unadjusted Cox proportional hazards regression model

c Adjusted for age, sex, race, and poverty status

d Adjusted for age, sex, race, poverty status, smoking status, body mass index, low density lipoprotein cholesterol, diagnosis of hypertension, and diagnosis of diabetes mellitus

e Linear trend test was performed using median of the quartile as a continuous variable in the Cox proportional hazards regression model 
Table 3 Stratified analysis of the association between red cell distribution width and all-cause mortality

\begin{tabular}{|c|c|c|c|}
\hline $\mathrm{RDW}^{\mathrm{a}}$ & $\mathrm{HR}^{\mathrm{b}}(95 \% \mathrm{Cl})$ & $\mathrm{HR}^{\mathbf{b}}(95 \% \mathrm{Cl})$ & P-interaction ${ }^{c}$ \\
\hline Sex & Men & Women & \\
\hline RDW-Q1 & 1.00 & 1.00 & 0.42 \\
\hline RDW-Q2 & $1.39(0.73-2.67)$ & $0.71(0.34-1.47)$ & \\
\hline RDW-Q3 & $1.20(0.60-2.38)$ & $0.85(0.42-1.7)$ & \\
\hline RDW-Q4 & $2.41(1.26-4.59)$ & $1.17(0.6-2.28)$ & \\
\hline P-trend ${ }^{d}$ & 0.006 & 0.29 & \\
\hline Race & African Americans & Whites & \\
\hline RDW-Q1 & 1.00 & 1.00 & 0.35 \\
\hline RDW-Q2 & $0.76(0.4-1.44)$ & $1.68(0.79-3.59)$ & \\
\hline RDW-Q3 & $0.86(0.46-1.58)$ & $1.57(0.71-3.49)$ & \\
\hline RDW-Q4 & $1.18(0.66-2.1)$ & $3.60(1.68-7.72)$ & \\
\hline P-trend ${ }^{d}$ & 0.22 & 0.001 & \\
\hline Poverty status & Above & Below & \\
\hline RDW-Q1 & 1.00 & 1.00 & 0.17 \\
\hline RDW-Q2 & $1.62(0.76-3.49)$ & $0.85(0.45-1.61)$ & \\
\hline RDW-Q3 & $2.17(1.03-4.56)$ & $0.63(0.32-1.24)$ & \\
\hline RDW-Q4 & $2.80(1.33-5.92)$ & $1.33(0.74-2.4)$ & \\
\hline P-trend ${ }^{d}$ & 0.005 & 0.18 & \\
\hline Current smoking & No & Yes & \\
\hline RDW-Q1 & 1.00 & 1.00 & 0.42 \\
\hline RDW-Q2 & $1.31(0.61-2.81)$ & $1.04(0.56-1.94)$ & \\
\hline RDW-Q3 & $0.82(0.34-1.99)$ & $1.28(0.71-2.31)$ & \\
\hline RDW-Q4 & $1.83(0.82-4.06)$ & $1.82(1.03-3.23)$ & \\
\hline P-trend ${ }^{d}$ & 0.17 & 0.01 & \\
\hline BMI & $\mathrm{BMI}<25.0$ & $\mathrm{BMI} \geq 25.0$ & \\
\hline RDW-Q1 & 1.00 & 1.00 & 0.004 \\
\hline RDW-Q2 & $1.16(0.59-2.28)$ & $0.89(0.44-1.8)$ & \\
\hline RDW-Q3 & $0.79(0.37-1.69)$ & $1.34(0.7-2.55)$ & \\
\hline RDW-Q4 & $1.44(0.7-2.95)$ & $2.06(1.11-3.83)$ & \\
\hline P-trend ${ }^{d}$ & 0.39 & 0.002 & \\
\hline
\end{tabular}

$B M I$ body mass index, $\mathrm{Cl}$ confidence intervals, $H R$ hazard ratio

a $\mathrm{RDW}$, red cell distribution width levels in quartiles. Quartile cut-off points were $13.2,13.8$, and $14.6 \%$

${ }^{\mathrm{b}}$ Multivariable adjusted Cox proportional hazards regression model

c $p$-value from likelihood ratio test of interaction

${ }^{d}$ Linear trend test was performed using median of the quartile as a continuous variable in the Cox proportional hazards regression model

eGFR $[-0.005(-0.01,-0.001)]$ showed an inverse association with RDW (Additional file 1: Table S3).

\section{Novel determinants of RDW}

We identified a novel significant association between RDW and illicit drug use: marijuana use [yes vs. no; -0.3 $(-0.5,-0.03)$ ], current cocaine use [yes vs. no; -0.4 $(-0.7,-0.02)]$. WHR was positively associated with RDW [1.4 (0.2, 2.5)] (Table 4). Stratified analyses by sex, poverty status, and race showed that both marijuana $[\mathrm{b}$
Table 4 Association between red cell distribution width and waist-hip ratio, and illicit drug use

\begin{tabular}{|c|c|c|c|c|}
\hline Characteristics & Beta $^{a}$ & $95 \% \mathrm{Cl}$ & Beta $^{b}$ & $95 \% \mathrm{Cl}$ \\
\hline \multicolumn{5}{|c|}{ Overall population } \\
\hline WHR & 0.3 & $0.02,0.5$ & 1.4 & $0.2,2.5$ \\
\hline \multicolumn{5}{|l|}{ Marijuana use } \\
\hline No & Ref & & Ref & \\
\hline Yes & -0.2 & $-0.4,-0.01$ & -0.3 & $-0.5,-0.03$ \\
\hline \multicolumn{5}{|c|}{ Current cocaine use } \\
\hline No & Ref & & Ref & \\
\hline Yes & -0.3 & $-0.6,-0.02$ & -0.4 & $-0.7,-0.02$ \\
\hline \multicolumn{5}{|c|}{ Stratified analysis by sex } \\
\hline \multicolumn{5}{|l|}{ Men } \\
\hline WHR & 1.0 & $-0.1,2$ & 0.3 & $-1.4,2.1$ \\
\hline \multicolumn{5}{|l|}{ Marijuana use } \\
\hline No & Ref & & Ref & \\
\hline Yes & -0.04 & $-0.2,0.2$ & -0.002 & $-0.3,0.3$ \\
\hline \multicolumn{5}{|c|}{ Current cocaine use } \\
\hline No & Ref & & Ref & \\
\hline Yes & -0.02 & $-0.3,0.3$ & -0.1 & $-0.5,0.3$ \\
\hline \multicolumn{5}{|l|}{ Women } \\
\hline WHR & 0.2 & $-0.1,0.5$ & 1.6 & $0.1,3.2$ \\
\hline \multicolumn{5}{|l|}{ Marijuana use } \\
\hline No & Ref & & Ref & \\
\hline Yes & -0.3 & $-0.7,-0.01$ & -0.6 & $-1.0,-0.2$ \\
\hline \multicolumn{5}{|c|}{ Current cocaine use } \\
\hline No & Ref & & Ref & \\
\hline Yes & -0.6 & $-1.1,-0.1$ & -0.6 & $-1.2,-0.1$ \\
\hline \multicolumn{5}{|c|}{ Stratified analysis by race } \\
\hline \multicolumn{5}{|c|}{ African American } \\
\hline WHR & 2.5 & $1.2,3.7$ & 1.6 & $-0.1,3.4$ \\
\hline \multicolumn{5}{|l|}{ Marijuana use } \\
\hline No & Ref & & Ref & \\
\hline Yes & -0.2 & $-0.5,0.03$ & -0.3 & $-0.6,0.03$ \\
\hline \multicolumn{5}{|c|}{ Current cocaine use } \\
\hline No & Ref & & Ref & \\
\hline Yes & -0.3 & $-0.7,0.1$ & -0.3 & $-0.8,0.1$ \\
\hline \multicolumn{5}{|l|}{ White } \\
\hline WHR & 0.1 & $-0.1,0.3$ & 0.6 & $-0.7,2.0$ \\
\hline \multicolumn{5}{|l|}{ Marijuana use } \\
\hline No & Ref & & Ref & \\
\hline Yes & -0.1 & $-0.4,0.2$ & -0.2 & $-0.5,0.2$ \\
\hline \multicolumn{5}{|c|}{ Current cocaine use } \\
\hline No & Ref & & Ref & \\
\hline Yes & -0.3 & $-0.6,0.1$ & -0.4 & $-0.9,0.1$ \\
\hline \multicolumn{5}{|c|}{ Stratified analysis by poverty status } \\
\hline \multicolumn{5}{|c|}{ Below poverty } \\
\hline WHR & 1.8 & $0.4,3.2$ & 0.3 & $-1.5,2.1$ \\
\hline \multicolumn{5}{|l|}{ Marijuana use } \\
\hline No & Ref & & Ref & \\
\hline Yes & -0.3 & $-0.6,0.1$ & -0.3 & $-0.7,0.1$ \\
\hline
\end{tabular}


Table 4 continued

\begin{tabular}{lrlrl}
\hline Characteristics & Beta $^{\mathbf{a}}$ & $\mathbf{9 5 \%} \mathbf{C l}$ & Beta $^{\mathbf{b}}$ & $\mathbf{9 5 \%} \mathbf{C l}$ \\
\hline Current cocaine use & & & & \\
No & Ref & & Ref & \\
Yes & -0.5 & $-0.9,-0.1$ & -0.6 & $-1.1,-0.1$ \\
Above poverty & & & & \\
WHR & 0.2 & $-0.03,0.4$ & 2.1 & $0.7,3.6$ \\
Marijuana use & & & & \\
No & Ref & & Ref & \\
Yes & -0.1 & $-0.4,0.1$ & -0.3 & $-0.6,0.05$ \\
Current cocaine use & & & & \\
No & Ref & & Ref & \\
Yes & 0.01 & $-0.4,0.4$ & 0.03 & $-0.5,0.5$ \\
\hline
\end{tabular}

Cl confidence interval, WHR waist-hip ratio

a Adjusted for age, sex, race, and poverty status

b Full model adjusted for age, sex, race, poverty status, current cigarette smoking, body mass index, diagnosis of hypertension, diagnosis of diabetes mellitus, low density lipoprotein cholesterol, estimated glomerular filtration rate, and high-sensitivity C-reactive protein

$(95 \% \mathrm{CI})=-0.6(-1.0,-0.2)]$, and cocaine use $[\mathrm{b}(95 \%$ $\mathrm{CI})=-0.6(-1.2,-0.1)]$ were associated with RDW among women, and the effect of current cocaine use was limited to participants below poverty level [b (95\% CI $)=-0.6(-1.1,-0.2)]($ Table 4$)$.

\section{Gene $\times$ environment interaction in RDW}

After correcting for multiple testing using the Bonferroni method, there was no evidence of gene $\times$ environment interaction between RDW linked genetic variants (TMEM57-RHD-rs10903129, NOL4L-rs4911241, CD36-rs3211938, LINC01184-SLC12A2-rs10063647, LINC01184-SLC12A2-rs10089, TRIB1-rs2954029) and non-genetic factors previously known to influence RDW (Additional file 1: Table S4).

\section{Discussion}

In the present study, using prospectively collected clinical, genetic and mortality data, we assessed the association between RDW and all-cause and disease-specific mortality risk, as well as investigated determinants of RDW. Elevated RDW conferred a 1.73-times increased risk of all-cause mortality and a 2.49-times increased risk of CVD-specific mortality in urban adults. This elevated risk was substantially modified by BMI. Our results confirmed previously reported predictors of RDW such as cigarette smoking, BMI and CRP, and identified novel associations between RDW and illicit drug use: marijuana and current cocaine use, and WHR.
Our findings of positive associations between RDW and premature mortality among younger urban adults are consistent with previous reports of elevated RDW and all-cause and CVD-specific mortality among middle and older age individuals. Two separate studies using the National Health and Nutrition Examination Survey (NHANES) (1988-1994) data reported that the highest quantile of RDW was associated with a 2-fold, and 2.1to 2.3-fold increased risk of all-cause and CVD-specific mortality, respectively $[9,10]$. In a meta-analysis of RDW and mortality in older individuals, Patel et al. reported a positive association between RDW and all-cause and cause specific mortalities due to CVD, cancer, and other causes [11]. It should be noted that our cohort represents adults younger (mean age $=48.1$ years) than participants included in the NHANES study (mean age $=62.0$ years) [9], and the meta-analysis (mean age ranged 73.679.1 years) [11]. Also, the presents study contains a large proportion of AAs (57\%) compared to $16.9 \%$ in previous studies of RDW and mortality [11].

Contrary to the declining mortality rate trends seen in industrialized countries, the recent rise of premature mortality rates among US adults in their prime-age is a serious clinical and public health problem [18]. Most of these early deaths, measured as years of life lost, were due mainly to preventable factors such as ischemic heart disease, drug and alcohol use disorders which are themselves multifactorial in origin $[18,19,28]$. While some of these factors (socioeconomic factors, tobacco use, drug and alcohol use, sedentary lifestyle) have long been the focus of clinical and public health prevention strategies, there is a substantial disparity in premature mortality, particularly among young vulnerable and disadvantaged individuals $[16,17,29]$. To improve targeted and precise interventions and eliminate this disparity gap, identifying readily available and less expensive biomarkers of premature mortality is important. The fact that RDW is simple and routinely measured clinical parameter makes it a useful tool for health prevention intervention strategies.

RDW reflects variations in red blood cell size (anisocytosis). In addition to aiding the clinical evaluation of anemia, RDW is independently associated with chronic disease risk and mortality [6]. The exact pathophysiological mechanisms behind this association are not clearly understood although inflammatory cytokines, oxidative stress, and neurohumoral factors are implicated as potential mediators $[2,9,12,30]$. Inflammatory cytokines such as interleukin-1, tumor necrosis factor- $\alpha$, and interferon- $\gamma$, which are known to be released in chronic inflammatory states, could affect bone marrow red blood cell production, maturation, and could subsequently 
lead to anisocytosis [31]. Although we found a positive association between RDW and inflammation markers, it should be noted that the effect of RDW on both allcause and cardiovascular mortality risk was independent of hsCRP and ESR - two commonly used markers of systemic inflammation. On the other hand, oxidative stress and systemic inflammation have a complex interrelationship and they are associated with morbidity and mortality. A recent study conducted in elderly individuals showed that serum oxidative stress markers (e.g., derivatives of reactive oxygen metabolites and total thiol levels) were significantly associated with all-cause mortality [32]. However, following adjustment for CRP, the effect of derivatives of reactive oxygen metabolites on mortality was no longer significant. Further, the positive correlation between derivatives of reactive oxygen metabolites and CRP suggested the effect of these non-specific markers of oxidative stress might partly be explained by inflammation [32]. To date, there are no studies that directly investigated oxidative stress and RDW in relation to mortality risk and thus we cannot rule out that the effect of increased RDW on mortality is not mediated through oxidative stress processes. To understand the role of SCT on the association between RDW and mortality risk, we assessed the confounding effect of SCT and interaction between SCT and RDW. We found no evidence of a main effect, interaction or confounding effect for SCT. Together with the results of model calibration analyses, these data indicate that RDW could be a valuable predictive biomarker of mortality to identify not only older individuals but also younger adults at higher risk of premature mortality. Future studies utilizing red blood cell specific oxidative stress markers such as fluorescent heme degradation products [33] which are not associated with CRP [34] could shed light on the causal link between RDW, oxidative stress and mortality risk.

The findings in the present study that sex, race, cigarette smoking, BMI, and hsCRP influence RDW are consistent with previous reports $[6,7,12,13]$. In the present study, we provide the first evidence that WHR and illicit drug use as novel independent determinants of RDW. The positive association between WHR and RDW independent of BMI and other factors known to influence RDW suggests that central obesity as novel predictor of RDW, and could shine light as to how RDW is associated with chronic disease morbidity and mortality.

Interestingly, we found inverse associations between marijuana and cocaine use and RDW independent of potential confounders including systemic inflammation markers. There are no experimental data on the link between RDW, cocaine, and $\Delta^{9}$-tetrahydrocannabinol (THC). In humans, little is known about the effect of illicit drug use on red blood cell physiology. Two small studies that assessed the effect of cocaine on bone marrow function reported conflicting findings. A study by Siegel et al. showed cocaine use resulted in erythrocytosis while another study by Weber et al. found no significant association between cocaine use and red blood cell count as well as reticulocyte count suggesting that cocaine use might not have effect on bone marrow mediated erythropoiesis [35, 36]. Cocaine use is known to have negative effects including raised blood pressure and oxidative stress damage to cardiac tissues [37]. Although cannabinoids regulate the production of inflammatory mediators [38] and sometimes prescribed by physicians for therapeutic benefits, recent reports indicate that marijuana (natural or synthetic toxic street form) is associated with production of reactive oxygen species, and cardiovascular diseases [39]. These findings imply that, in the context of the current drug use epidemic, the significance of RDW as a marker of mortality requires additional evaluation. Further experimental studies are required to elucidate the molecular mechanism behind illicit drug use and RDW. We did not find a significant gene $\mathrm{x}$ environment interaction in our targeted analysis after correcting for multiple testing. This could be due to the smaller sample size with genotype data. Future interaction studies on larger samples using genome-wide sequence variants are required to understand the influence of environmental exposure on genes that affect RDW levels.

The limitations of the present study include the following: first, the lack of genotype data among white participants that precluded gene $\times$ environment interaction analysis. Second, the absence of objectively measured data in our cohort on potential confounders such as serum nutrients and antioxidants, although a previous study showed serum antioxidants did not change the effect of RDW on mortality [9]. Third, the smaller number of subjects with specific causes of death limited further disease-specific mortality analyses.

The strengths of this study are its large sample size, inclusion of participants with diverse race and socioeconomic status, and availability of a wide array of data on epidemiologic, environmental, clinical (e.g., highsensitivity CRP) and genomic data. Our results should be applicable to similar urban dwelling communities. Compared to the NHANES study which was collected between 1988 and 1994 and included rural and urban communities, our study used recently collected data (2004-2013), and was comprised of younger adults from a single urban study site, minimizing unmeasured confounding by geographic localities. Previous studies of RDW were conducted mostly in the elderly, and individuals of European ancestry [11]. By focusing on racially diverse younger individuals who are at risk of health 
disparity, we showed that the adverse effects of elevated RDW could be seen earlier in the life course.

\section{Conclusions}

In summary, RDW is strongly associated with mortality in urban AA and white adults that was modified by obesity. Our findings have several implications: first, RDW is an attractive target for intervention strategies as it is simple and readily available. Second, RDW could be used to identify vulnerable population groups with substantial burdens of premature death for precise and tailored interventions on modifiable factors of mortality. Clinical and public health prevention strategies could benefit from incorporating emerging biomarkers of mortality such as RDW. Third, during physician office visits, it provides an excellent opportunity for health care providers for individualized assessment, and to look out for preventable and/or treatable causes of premature mortality in subjects with elevated RDW. Because RDW is already included in complete blood count, it would not incur any additional cost for testing unlike other serum biomarkers of disease risk. Our results strengthen the call to broaden the use of RDW beyond its conventional use in the management of anemia $[1,6]$. Further research is required to extend our findings, identify best approaches to target RDW, and evaluate its utility in public health surveillance using randomized controlled trials.

\section{Additional file}

Additional file 1. Additional Figure S1 and Tables S1-S4.

\section{Abbreviations}

AA: African American; BMI: body mass index; CES-D: Center for Epidemiologic Studies-Depression scale; Cl: confidence interval; CVD: cardiovascular disease; ESR: erythrocyte sedimentation rate; eGFR: estimated glomerular filtration rate HANDLS: Healthy Aging in Neighborhoods of Diversity across the Life Span study; HR: hazard ratio; hsCRP: high-sensitivity C-reactive protein; ICD: International Classification of Diseases; LDL: low density lipoprotein cholesterol; NDI: National Death Index database; RDW: red cell distribution width;WBC: white
\end{abstract} blood cell count; WHR: waist-hip ratio.

\section{Authors' contributions}

MKE and ABZ acquired funding and are co-principal investigators of the study. SMT conducted all statistical analyses. MAN participated in acquisition of genetic data. All authors contributed to writing and revision of the manuscript, and approved the final version for publication. All authors read and approved the final manuscript.

\section{Author details \\ ${ }^{1}$ Laboratory of Epidemiology and Population Sciences, National Institute on Aging, National Institutes of Health, Room \# 04C222, Suite 100, 251 Bayview Boulevard, Baltimore, MD 21224, USA. ${ }^{2}$ Laboratory of Neurogenetics, National Institute on Aging, National Institutes of Health, Bethesda, MD 20892 USA. ${ }^{3}$ Data Tecnica International LLC, Glen Echo, MD 20812, USA.}

\section{Acknowledgements}

We would like to thank study participants, Dr. Ngozi Ejiogu and the HANDLS medical staff for the excellent medical evaluations of all participants.

\section{Competing interests}

MAN is supported by a consulting contract between Data Tecnica International LLC and the National Institute on Aging (NIA), National Institutes of Health (NIH), Bethesda, MD, USA. MAN consults for Illumina Inc., the Michael J. Fox Foundation, and the University of California Healthcare. The rest of the authors declare that they have no competing interests.

\section{Availability of data and materials}

Descriptions of data available in the HANDLS study including the current study can be found study's website (https://handls.nih.gov/). The dataset used during the current study are available from the corresponding author on reasonable request.

\section{Consent for publication}

All study participants provided a signed written informed consent. All authors who have contributed to this work approved its publication in the Journal of Translational Medicine.

\section{Ethics approval and consent to participate}

This study was approved by the institutional review board of the National Institutes of Environmental Health Sciences, National Institutes of Health. The study Protocol Number is 09-AG-N248. All study participants provided a signed written informed consent.

\section{Funding}

This study was supported by the Intramural Research Program of the National Institute on Aging (NIA), National Institutes of Health (NIH), Project\# Z01-AG000513, Protocol\# 09-AG-N248.

\section{Publisher's Note}

Springer Nature remains neutral with regard to jurisdictional claims in published maps and institutional affiliations.

Received: 15 June 2017 Accepted: 6 October 2017

Published online: 13 October 2017

\section{References}

1. Patel A, Brett SJ. Identifying future risk from routine tests? Crit Care Med. 2014;42:999-1000.

2. Felker GM, Allen LA, Pocock SJ, Shaw LK, McMurray JJ, Pfeffer MA, Swedberg K, Wang D, Yusuf S, Michelson EL, et al. Red cell distribution width as a novel prognostic marker in heart failure: data from the CHARM Program and the Duke Databank. J Am Coll Cardiol. 2007;50:40-7.

3. Lappegard J, Ellingsen TS, Vik A, Skjelbakken T, Brox J, Mathiesen EB, Johnsen SH, Braekkan SK, Hansen JB. Red cell distribution width and carotid atherosclerosis progression. The Tromso Study. Thromb Haemost. 2015;113:649-54.

4. Emans ME, Gaillard CA, Pfister R, Tanck MW, Boekholdt SM, Wareham NJ, Khaw KT. Red cell distribution width is associated with physical inactivity and heart failure, independent of established risk factors, inflammation or iron metabolism; the EPIC-Norfolk study. Int J Cardiol. 2013;168:3550-5.

5. Zoller B, Melander O, Svensson P, Engstrom G. Red cell distribution width and risk for venous thromboembolism: a population-based cohort study. Thromb Res. 2014;133:334-9.

6. Salvagno GL, Sanchis-Gomar F, Picanza A, Lippi G. Red blood cell distribution width: a simple parameter with multiple clinical applications. Crit Rev Clin Lab Sci. 2015;52:86-105.

7. Zalawadiya SK, Veeranna V, Panaich SS, Afonso L. Red cell distribution width and risk of peripheral artery disease: analysis of national health and nutrition examination survey 1999-2004. Vasc Med. 2012;17:155-63.

8. Purtle SW, Moromizato T, MCKane CK, Gibbons FK, Christopher KB. The association of red cell distribution width at hospital discharge and out-ofhospital mortality following critical illness. Crit Care Med. 2014;42:918-29.

9. Patel KV, Ferrucci L, Ershler WB, Longo DL, Guralnik JM. Red blood cell distribution width and the risk of death in middle-aged and older adults. Arch Intern Med. 2009;169:515-23. 
10. Perlstein TS, Weuve J, Pfeffer MA, Beckman JA. Red blood cell distribution width and mortality risk in a community-based prospective cohort. Arch Intern Med. 2009;169:588-94.

11. Patel KV, Semba RD, Ferrucci L, Newman AB, Fried LP, Wallace RB, Bandinelli S, Phillips CS, Yu B, Connelly S, et al. Red cell distribution width and mortality in older adults: a meta-analysis. J Gerontol A Biol Sci Med Sci. 2010;65:258-65.

12. Lippi G, Targher G, Montagnana M, Salvagno GL, Zoppini G, Guidi GC. Relation between red blood cell distribution width and inflammatory biomarkers in a large cohort of unselected outpatients. Arch Pathol Lab Med. 2009:133:628-32.

13. Vaya A, Alis R, Hernandez-Mijares A, Sola E, Camara R, Rivera L, Romagnoli M, Laiz B. Red blood cell distribution width is not related with inflammatory parameters in morbidly obese patients. Clin Biochem. 2014;47:464-6.

14. Chami N, Chen MH, Slater AJ, Eicher JD, Evangelou E, Tajuddin SM, LoveGregory L, Kacprowski T, Schick UM, Nomura A, et al. Exome genotyping identifies pleiotropic variants associated with red blood cell traits. Am J Hum Genet. 2016;99:8-21.

15. Chen Z, Tang H, Qayyum R, Schick UM, Nalls MA, Handsaker R, Li J, Lu Y, Yanek LR, Keating B, et al. Genome-wide association analysis of red blood cell traits in African Americans: the COGENT Network. Hum Mol Genet. 2013:22:2529-38.

16. Zonderman AB, Mode NA, Ejiogu N, Evans MK. Race and poverty status as a risk for overall mortality in community-dwelling middle-aged adults. JAMA Intern Med. 2016;176:1394-5.

17. Stringhini S, Carmeli C, Jokela M, Avendano M, Muennig P, Guida F, Ricceri F, d'Errico A, Barros H, Bochud M, et al. Socioeconomic status and the $25 \times 25$ risk factors as determinants of premature mortality: a multicohort study and meta-analysis of 1.7 million men and women. Lancet. 2017;389(10075):1229-37.

18. Case A, Deaton A. Rising morbidity and mortality in midlife among white non-Hispanic Americans in the 21st century. Proc Natl Acad Sci USA. 2015:112:15078-83.

19. Global Action Plan for the Prevention and Control of noncommunicable diseases 2013-2020. [http://www.who.int/nmh/events/ncd_action_plan/ en/]. Accessed 7 June 2017.

20. Evans MK, Lepkowski JM, Powe NR, LaVeist T, Kuczmarski MF, Zonderman AB. Healthy aging in neighborhoods of diversity across the life span (HANDLS): overcoming barriers to implementing a longitudinal, epidemiologic, urban study of health, race, and socioeconomic status. Ethn Dis. 2010;20:267-75.

21. Radloff LS. The CES-D scale: a self-report depression scale for research in the general population. Appl Psychol Meas. 1977;1:385-401.

22. Inker LA, Schmid CH, Tighiouart H, Eckfeldt JH, Feldman HI, Greene T, Kusek JW, Manzi J, Van Lente F, Zhang YL, et al. Estimating glomerular filtration rate from serum creatinine and cystatin C. N Engl J Med. 2012;367:20-9.

23. Mode NA, Evans MK, Zonderman AB. Race, neighborhood economic status, income inequality and mortality. PLoS ONE. 2016;11:e0154535.

24. Purcell S, Neale B, Todd-Brown K, Thomas L, Ferreira MA, Bender D, Maller J, Sklar P, de Bakker PI, Daly MJ, Sham PC. PLINK: a tool set for wholegenome association and population-based linkage analyses. Am J Hum Genet. 2007:81:559-75.
25. Thiebaut AC, Benichou J. Choice of time-scale in Cox's model analysis of epidemiologic cohort data: a simulation study. Stat Med. 2004;23:3803-20.

26. Demler OV, Paynter NP, Cook NR. Tests of calibration and goodness-of-fit in the survival setting. Stat Med. 2015:34:1659-80.

27. R Core Team (2015). R: A language and environment for statistical computing. R Foundation for Statistical Computing, Vienna, Austria. https:// wWw.R-project.org/.

28. Collaborators GMaCoD. Global, regional, and national life expectancy, all-cause mortality, and cause-specific mortality for 249 causes of death, 1980-2015: a systematic analysis for the Global Burden of Disease Study 2015. Lancet. 2015;388:1459-544.

29. Chetty R, Stepner M, Abraham S, Lin S, Scuderi B, Turner N, Bergeron A, Cutler D. The association between income and life expectancy in the United States, 2001-2014. JAMA. 2016;315:1750-66.

30. Fukuta H, Ohte N, Mukai S, Saeki T, Asada K, Wakami K, Kimura G. Elevated plasma levels of B-type natriuretic Peptide but not $C$-reactive protein are associated with higher red cell distribution width in patients with coronary artery disease. Int Heart J. 2009;50:301-12.

31. Macdougall IC, Cooper A. The inflammatory response and epoetin sensitivity. Nephrol Dial Transplant. 2002;17(Suppl 1):48-52.

32. Schottker B, Saum KU, Jansen EH, Boffetta P, Trichopoulou A, Holleczek B, Dieffenbach AK, Brenner H. Oxidative stress markers and all-cause mortality at older age: a population-based cohort study. J Gerontol A Biol Sci Med Sci. 2015;70:518-24.

33. Jacob KD, Noren Hooten N, Trzeciak AR, Evans MK. Markers of oxidant stress that are clinically relevant in aging and age-related disease. Mech Ageing Dev. 2013;134:139-57.

34. Szanton SL, Rifkind JM, Mohanty JG, Miller ER 3rd, Thorpe RJ, Nagababu E, Epel ES, Zonderman AB, Evans MK. Racial discrimination is associated with a measure of red blood cell oxidative stress: a potential pathway for racial health disparities. Int J Behav Med. 2012;19:489-95.

35. Siegel AJ, Sholar MB, Mendelson JH, Lukas SE, Kaufman MJ, Renshaw PF, MCDonald JC, Lewandrowski KB, Apple FS, Stec JJ, et al. Cocaine-induced erythrocytosis and increase in von Willebrand factor: evidence for drug-related blood doping and prothrombotic effects. Arch Intern Med. 1999:159:1925-9.

36. Weber JE, Larkin GL, Boe CT, Fras A, Kalaria AS, Maio RF, Luchessi B, Ensign $L$, Sweeney B, Hollander JE. Effect of cocaine use on bone marrow-mediated erythropoiesis. Acad Emerg Med. 2003;10:705-8.

37. Cerretani D, Fineschi V, Bello S, Riezzo I, Turillazzi E, Neri M. Role of oxidative stress in cocaine-induced cardiotoxicity and cocaine-related death. Curr Med Chem. 2012;19:5619-23.

38. Klein TW. Cannabinoid-based drugs as anti-inflammatory therapeutics. Nat Rev Immunol. 2005;5:400-11.

39. Wolff $\vee$, Rouyer O, Geny B. Adverse health effects of marijuana use. N Engl Med. 2014;371:878.

\section{Submit your next manuscript to BioMed Central and we will help you at every step:}

- We accept pre-submission inquiries

- Our selector tool helps you to find the most relevant journal

- We provide round the clock customer support

- Convenient online submission

- Thorough peer review

- Inclusion in PubMed and all major indexing services

- Maximum visibility for your research

Submit your manuscript at www.biomedcentral.com/submit
C BioMed Central 